\title{
Computer Aided Analysis for Biosensing and Screening
}

\author{
Xiang-Ming Li, Bernard S. Liang, and Henry Y. Wang \\ Department of Chemical Engineering, University of Michigan, \\ Ann Arbor, Michigan 48109
}

Accepted for publication February 10, 1987

Biosensors with animal and microbial cells immobilized close to the tip of a membrane electrode háve been developed for chemical and drug testing. Our experimental results show that biosensors can be used for drug screening and to provide useful information about various cell-chemical interactions. A computer aided analysis (CAA) software package is being developed here using the biosensor for various screening purposes. This software package enables us to use a computer to analyze the biosensor dynamic responses. Computer simulation and parameter estimation techniques are used to select the best model and to describe the biochemical and pharmacologic effects of various chemicals and drugs on different cell lines.

\section{INTRODUCTION}

Every year, many chemicals are tested for toxicity and other properties using biological assays. These tests include antibiotic and antitumor agent screening. Conventional biological assays, such as agar diffusion and viable cell count, are very labor intensive and are difficult to automate. Semi-automation using image analyses to analyze the conventional bioassay results has been developed. A more revolutionary method is to use biosensors with various biological materials and transducers as tools for biological testing and screening.

Biosensors usually consist of two parts. One is the biomaterial which can interact with the chemicals being tested and produce a signai. The second part is an electrochemical or physical transducer which can convert the signal produced by the bio-material into potentiometric or amperometric signals. These signals can be processed automatically using a computer. The technology of biosensors has been reviewed by Lowe ${ }^{1}$ and others. Biosensors have been developed to test various chemicals such as nystatin ${ }^{2}$ and arginine. ${ }^{3}$ So far, the main concern for biosensor development is to relate the biosensor output information to the chemical concentration. In this work, we use the biosensor as a model microreactor to study various cell-chemical interactions. Immobilized whole cells, such as mouse leukemia cells (L1210), human foreskin fibroblast cells (HFF), and yeast, are the bio-materials in our biosensors. The electrochemical transducer is a modified Borkowski-
Johnson dissolved oxygen electrode. ${ }^{4}$ A schematic diagram of the biosensor is shown in Figure 1. Mechlorethamine $\mathrm{HCl}$, Methylglyoxal-bisguanylhydrazone (MGBG), sodium azide, and nystatin are the model drugs and chemicals that were used in this research.

The biosensor output, usually an electrical signal, is an indirect measurement of the cellular metabolism. These data need to be interpreted using a computer. We have developed a computer aided analysis (CAA) software package for data interpretation. In this software package, different kinetic models can be utilized during computer simulation. A parameter estimation technique is used to estimate the parameters in the kinetic models and to identify a suitable model based on the biosensor output. This

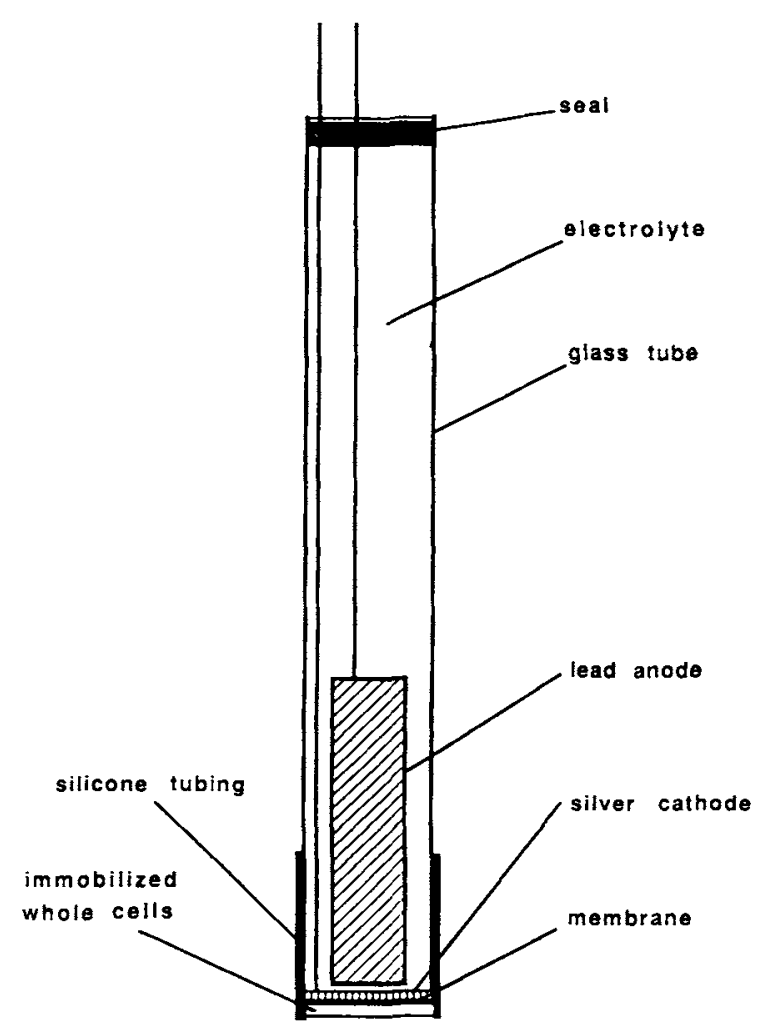

Figure 1. Schematic diagram of the biosensor. 
model and its parameters can then be used to evaluate the effects of various chemicals and drugs on different cell lines.

\section{EXPERIMENTAL WORK}

Two methods of immobilizing whole cells were used to construct the cell layer in the biosensor. The animal cells were immobilized using hydrogel, such as kappa carrageenan. A short piece of silicone tubing was placed at the tip of the biosensor and extended past the tip by about $2 \mathrm{~mm}$. The protrusion served as the gel-cell retainer for the gel-cell suspension. Kappa carrageenan gel $(4 \% \mathrm{~W} / \mathrm{V})$ was mixed with cells, such as mouse leukemia cell (L1210) and HFF cells, placed onto the tip of the D.O. electrode and allowed to solidify. The concentrations of L1210 and HFF in the gel were from $1-2 \times 10^{8} \mathrm{cell} / \mathrm{ml}$. Yeast cells were immobilized using filter paper. ${ }^{2}$ The yeast cells were adsorbed to a filter paper disk which was placed on the tip of the D.O. electrode and then covered with dialysis membrane (Spectrapor, M.W. cutoff: 12,000-14,000). The concentration of the yeast cell suspension was about $3 \times 10^{9} \mathrm{cell} / \mathrm{ml}$. A $0.5 \mathrm{mil}$ teflon membrane (Dupont FEP, Wilmington, DE) was used to cover the cathode and separate the cell layer from the electrolyte $(1 \mathrm{M} \mathrm{KOH})$.

The experimental apparatus is shown schematically in Figure 2. Two temperature controlled vessels $\left( \pm 0.1^{\circ} \mathrm{C}\right)$ were placed on magnetic stirrers. A step change of the solution cendition was realized by switching the biosensor from one vessel to the other. RPM1 1640 with $5 \%$ fetal bovine serum (FBS, Gibco) was used as medium for L1210 and other mammalian cell lines. Ringer's salt solution was used as the maintenance medium for yeast. More details of the experimental work was published in a separate report. ${ }^{5}$

Biosensors with L1210 and HFF cells were used for antitumor drug testing. During testing, the biosensor was placed in the maintenance medium saturated with sterile

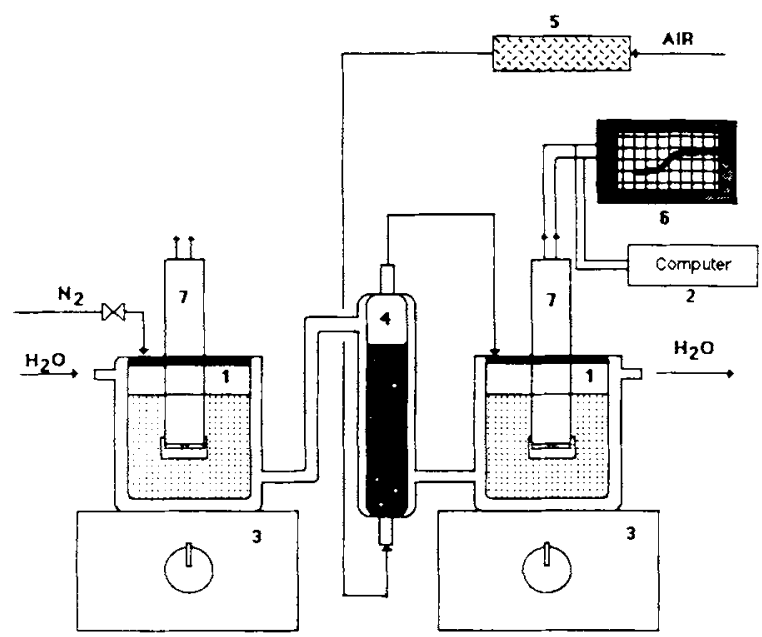

Figure 2. Schematic of biosensor experimental apparatus. Temperature controlled $\mathrm{H}_{2} \mathrm{O}$ is circulated through jacketed glassware: (1) testing vessel, (2) computer, (3) magnetic stirrer, (4) water saturator, (5) air filter, (6) chart recorder, and (7) biosensor. air. After the current output of the biosensor reached steady state, the test chemical was added to the medium. This is defined as time zero. The current output of the biosensor was recorded on a chart recorder or a microcomputer. Four current outputs using two cell lines (L1210, and HFF) and three concentrations of antitumor drug (Mechlorethamine $\mathrm{HCl}$ ) are shown in Figure 3. The gradual increases of the current output of the biosensors with L1210 imply a higher oxygen flux at the cathode surface, which indicates that the oxygen uptake of the cells decreased. This might be the result of a loss of cell viability (cytotoxic) or inhibition of cell respiration (cytostatic). The current output alone cannot distinguish between these mechanisms. Trypan blue staining and viable cell counts after testing, revealed that the tumor cells actually lost their viability. Therefore, the increase of current output of the biosensor can be related to the loss of viability of the tumor cells. The current output of the HFF biosensor did not demonstrate any increase when treated with Mechlorethamine $\mathrm{HCl}$. This indicates that HFF cells are not sensitive to the drug. The currenttime curves of the biosensors with $\mathrm{L} 1210$ in different chemical solutions, namely Mechlorethamine $\mathrm{HCl}$ and Methylglyoxal-bisguanylhydrazone (MGBG), using the same drug concentration $(36.6 \mathrm{mg} / \mathrm{L})$, are shown in Figure 4 . These results demonstrate that the biosensors can be used to test the cytotoxicity of various chemicals.

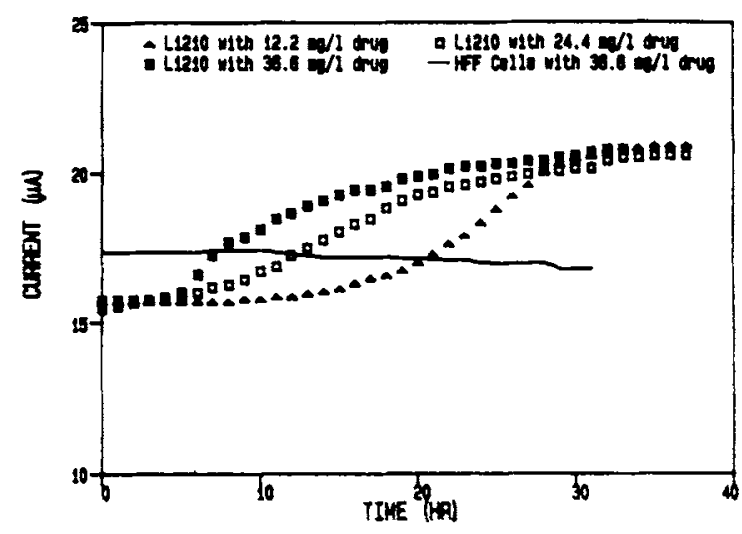

Figure 3. Biosensor response to mechlorethamine $\mathrm{HCl}$.

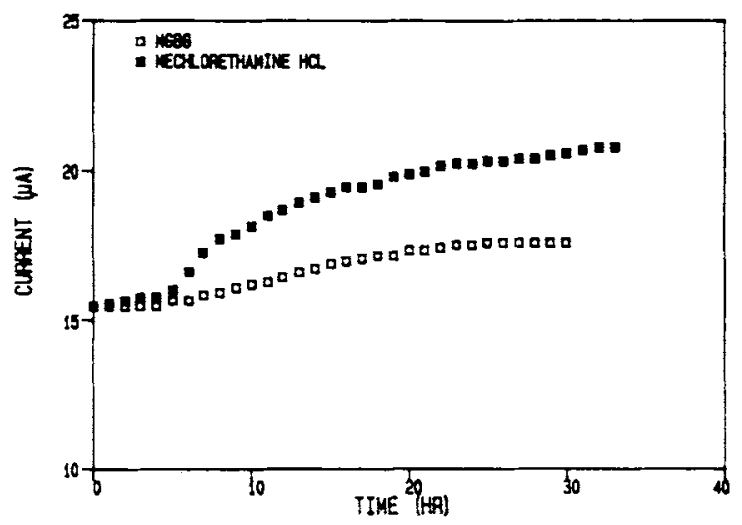

Figure 4. Comparison of Mechlorethamine $\mathrm{HCl}$ and MGBG effects on the biosensor with L1210. 
In order to discriminate between the cytotoxic and cytostatic effects, a different testing procedure was utilized. In this case, a yeast biosensor was used and first placed in a solution with the chemical to be tested. After a period of time, the biosensor was then switched to a medium without the chemical. Figures 5 and 6 show some interesting responses. The data in Figure 5 were obtained by testing sodium azide. The biosensor was placed in the solution with $5 \mathrm{mg} / \mathrm{L}$ sodium azide at time zero. The rapid rise of the current output demonstrated fast inhibition of the respiration of yeast. After $4500 \mathrm{~s}$, the biosensor was switched to a medium without sodium azide. The fast drop of the current output implies that the respiration of yeast cells was restored rapidly, and sodium azide had only cytostatic effects on yeast at this low concentration. Figure 6 shows the dynamic response of a biosensor with yeast cells to nystatin, an anti-fungal drug. At time zero, the biosensor was placed in the solution with a nystatin concentration of $25 \mathrm{mg} / \mathrm{L}$. The current output rose and reached steady state after $4 \mathrm{~h}$. After $4.48 \mathrm{~h}$, the biosensor was switched to a medium without nystatin. The current output did not drop, which indicated a cytotoxic effect of nystatin on yeast. From these current-time curves, one can see that it is fea-

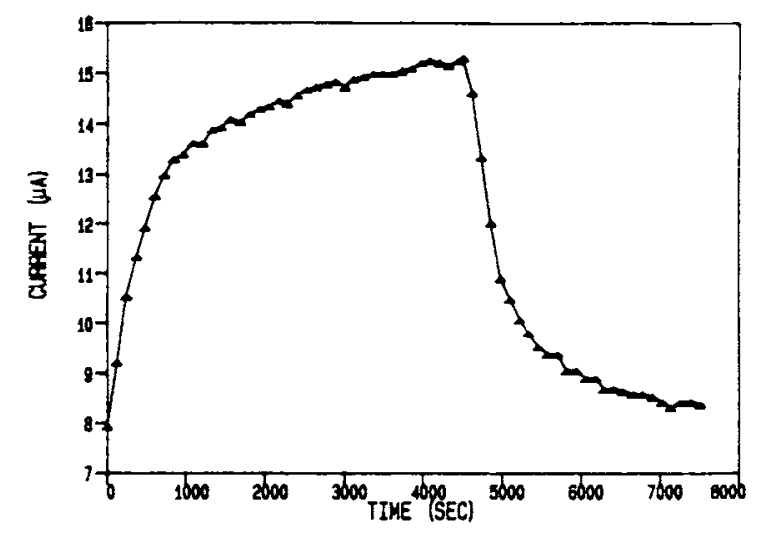

Figure 5. Response of yeast biosensor with sodium azide. At time $=0$, sodium azide was added to the medium. Concentration $=5 \mathrm{mg} / \mathrm{L}$. At time $=4500 \mathrm{~s}$, the biosensor was switched to fresh medium. The response showed cytostatic effect of sodium azide on yeast.

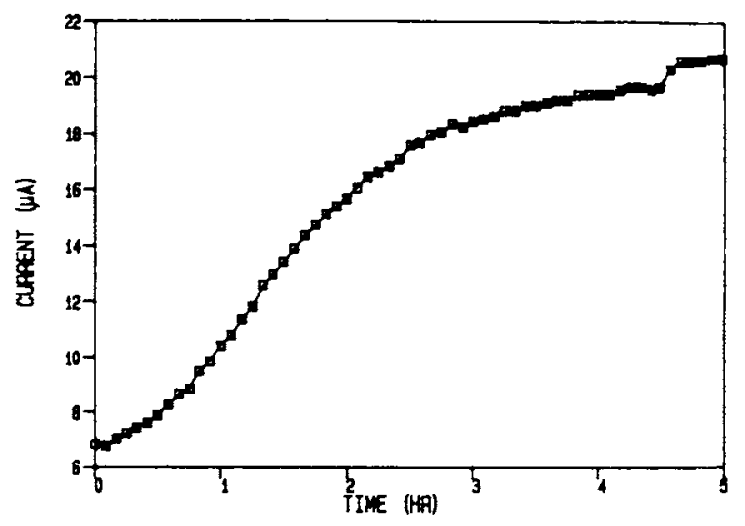

Figure 6. Response of yeast biosensor with nystatin. At time $=0$, nystatin was added to the medium. Concentration $=25 \mathrm{mg} / \mathrm{L}$. At time $=$ $4.48 \mathrm{~h}$, the biosensor was switched to fresh medium. The response showed cytotoxic effect of nystatin on yeast. sible to use these types of current-time curves directly to determine whether a chemical has a cytotoxic or a cytostatic effect on cells.

\section{COMPUTER AIDED ANALYSIS}

In order to interpret the biosensor responses, we have developed a Computer Aided Analysis (CAA) software package. This software for biosensing can help to select a suitable metabolic model for the cell-chemical interaction based on the biosensor responses. If the mathematical model is an algebraic equation or a differential equation that can be solved analytically, the parameters in the proposed model can be determined using various data plotting routines. The goodness of fit of the model is determined by comparing the predicted results to the experimental data. Since the mathematical model developed here cannot be solved analytically, computer simulation and parameter estimation techniques were used for model building and model discrimination. These techniques can help identify which metabolic model has the minimum error between the simulation results and experimental data. The governing processes at the tip of the biosensor are mass transfer and reaction, as shown schematically in Figure 7. During testing, dissolved oxygen diffuses through the boundary layer and reaches the immobilized cell layer. Part of the oxygen is consumed by the viable cells. The rest of the oxygen diffuses through the membrane and is reduced at the cathode surface. The current output of the dissolved oxygen (DO) electrode is dictated by the oxygen flux that reaches the cathode surface. If a chemical with a toxic or an inhibitory effect on the cells is present in the solution, it can diffuse into the cell layer and interact with the cells. Killing the viable cells or inhibiting their respiratory activities will reduce the oxygen uptake in the cell layer. Conse-

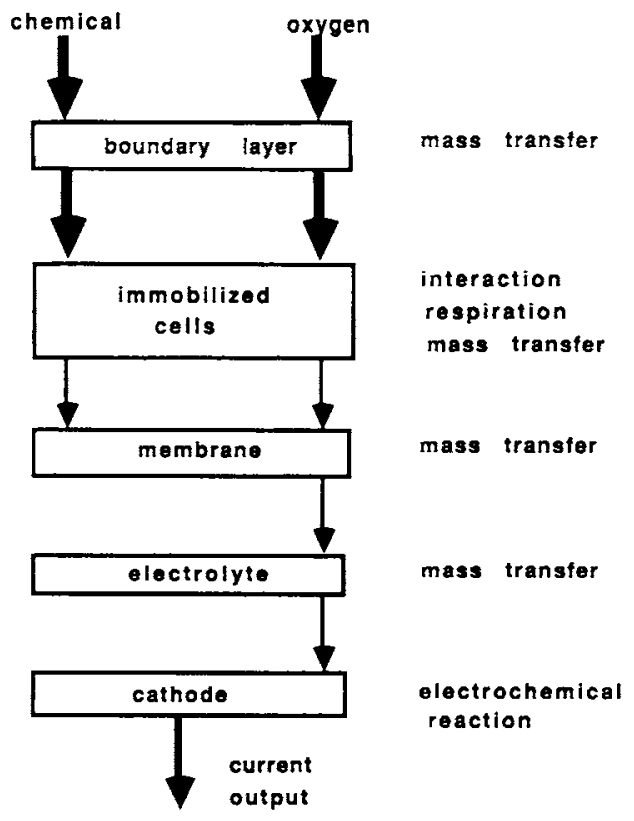

Figure 7. Mass transfer and reaction processes at the tip of the biosensor. 
quently, the oxygen flux to the cathode surface will increase thereby generating an increase in the current output of the DO electrode. By monitoring the current output of the electrode, the interaction between the chemical and the cells can be detected continuously.

The cell-chemical interaction, respiration of the cells, and transport processes all have an effect on the current output. A mathematical model was developed to relate these individual effects to the current output of the biosensor. The basic model we used is a four-layer model (the system geometry). Different metabolic models for the interaction of the chemical with the cells can be substituted into the overall model. By using a parameter estimation technique and the biosensor output, the computer can estimate the key parameters in each model and identify the model with the least error. The preliminary modeling work has been described. ${ }^{6}$ Since then, we have obtained more experimental data and improved our models. Different kinetic models with meaningful utility have been incorporated into the software and can be chosen on command.

The four-layer model and its coordinate system is shown in Figure 8. These four layers are: 1) electrolyte layer, 2) Teflon membrane, 3) cell layer, and 4) mass transfer boundary layer. By using mass balances and rate laws for each layer, the following governing equations were derived.

The oxygen balance equations are

$$
\begin{array}{rrr}
\frac{\partial C_{i}}{\partial t}=D_{i} \frac{\partial^{2} C_{i}}{\partial x^{2}}, \quad x_{i-1} \leq x \leq x_{i} & \text { for } i=1,2,4 \\
\frac{\partial C_{3}}{\partial t}=D_{3} \frac{\partial^{2} C_{3}}{\partial x^{2}}-\frac{\mu_{\max } C_{3}}{k_{m}+C_{3}} N, & x_{2} \leq x \leq x_{3}
\end{array}
$$

Equation (1) is Fick's second law applied to the electrolyte, the membrane, and the boundary layers. Equation (2) is an oxygen balance equation in gel-cell layer, in which a Michaelis-Menten type equation is used to describe the oxygen uptake of the viable cells.

The boundary conditions are

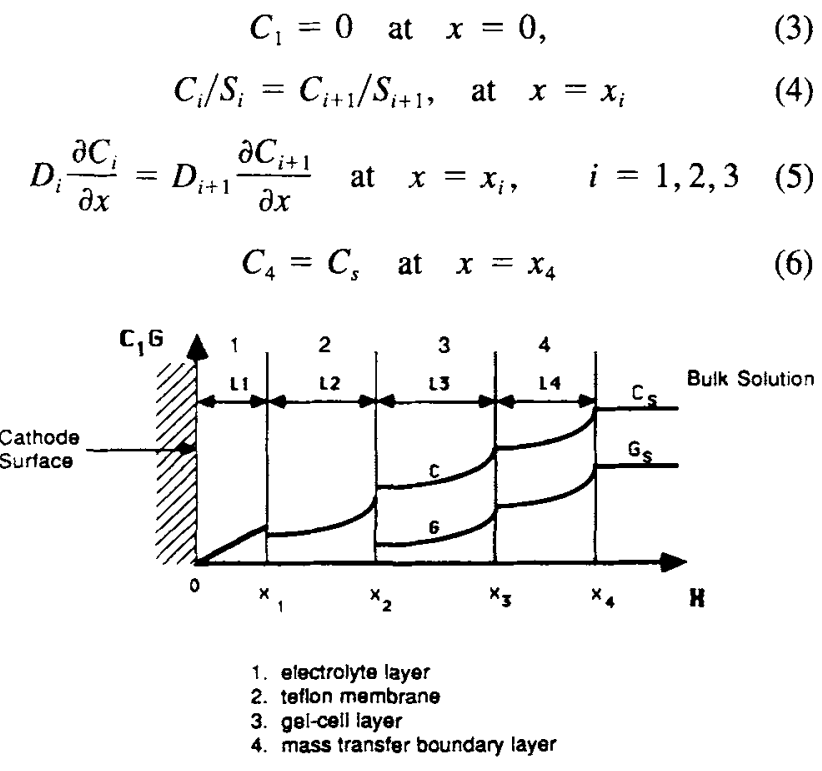

Figure 8. Schematic diagram of the four-layer model.
In eq. (3), the oxygen concentration is assumed to be zero at the cathode surface. Equations (4) and (5) are equilibrium and mass conservation conditions at the boundaries between adjacent layers. In eq. (6), the oxygen concentration at the outer surface of the boundary layer is assumed to be the oxygen concentration in the bulk solution. The initial conditions are

$$
\begin{gathered}
C_{i}=0, \quad i=1,2,3 \text { at } t=0 \\
C_{4}=C_{s} \text { at } t=0
\end{gathered}
$$

Equations (7) and (8) describe the initial oxygen concentrations in these different layers when the biosensor is placed in the solution.

The chemical (or drug) balance equations are

$$
\begin{aligned}
\frac{\partial G_{3}}{\partial t}=D_{i, 3} \frac{\partial^{2} G_{3}}{\partial x^{2}}-[\text { chemical deactivation rate }], & x_{2} \leq x \leq x_{3} \\
\frac{\partial G_{4}}{\partial t}=D_{t, 4} \frac{\partial^{2} G_{4}}{\partial x^{2}}, & x_{3} \leq x \leq x_{4}
\end{aligned}
$$

Equations (9) and (10) are mass balance equations for the chemical in the gel-cell layer and the boundary layer. In eq. (9), a general chemical deactivation term is utilized. Different kinetic model can be substituted in this term. These models will be displayed later.

The boundary conditions are

$$
\begin{gathered}
D_{t, 3} \frac{\partial G_{3}}{\partial t}=0 \quad \text { at } \quad x=x_{2} \\
G_{3}=G_{4} \quad \text { at } \quad x=x_{3} \\
D_{t, 3} \frac{\partial G_{3}}{\partial x}=D_{t, 4} \frac{\partial G_{4}}{\partial x} \quad \text { at } \quad x=x_{3} \\
G_{4}=G_{3} \quad \text { at } \quad x=x_{4}
\end{gathered}
$$

Equation (11) describes the impermeability of the chemical at the outer surface of the teflon membrane. Equations (12)-(14) are equilibrium and mass conservation conditions of the chemical at the surfaces between adjacent layers. The intermediate conditions are

$$
G_{i}=0, \quad i=3,4 \text { at } t=0
$$

The viable cell balance equation is

$$
\frac{d N}{d t}=-\left[\text { death rate of cells], } \quad x_{2} \leq x \leq x_{3}\right.
$$

In eq. (16), a nongrowth model with a general term of death rate is used to describe the viable cell balance. Different metabolic models can be adapted for this term. The intermediate conditions are

$$
N=N_{0} \quad \text { at } \quad t=0
$$

The current output equation is

$$
i=-4 F A D_{1} \frac{\partial C_{1}}{\partial x} \text { at } x=0
$$


Several metabolic models for chemical deactivation and cell death rate are listed as follows. The chemical (or drug) deactivation rate models are

$$
\begin{gathered}
k_{d} C^{n} N ; \\
k_{d} C^{n} t^{m} N ; \quad \text { and } \\
k_{d} \exp (n C) t^{m} N .
\end{gathered}
$$

The cell death rate models are

$$
k_{s} C^{n} N
$$

$$
0 \text { for } t C<T C_{0} ; \quad k_{s} C^{n} t^{m} N \quad \text { for } t C \geq T C_{0}
$$

$$
k_{s} \exp (n C) t^{m} N \text {. }
$$

These are nonstructured models. Some of them, such as the power law with $m=1$ and $n=1$, have been used in modeling disinfection. ${ }^{7}$ These models, except model (1), all include a time variable. This variable can be related to the accumulation of the chemical inside the cells. In model (2) of the cell death rate, a critical product of time and concentration, $T C_{0}$, has been defined. It means that before this product reaches a critical value, the cells will not be killed. A higher chemical concentration will yield a shorter cell death lag phase. These kinetic models can be incorporated into the overall model.

The computer simulation is based on numerical solution of these nonlinear differential equations using a finite difference method. For a given deactivation model, cell death model, and set of operating conditions, the computer solves the equations to produce a simulated biosensor output. In all of these models there are some unknown parameters, such as $\mu_{\max }$ and $k_{s}$. They can be estimated by using a parameter estimation routine. A golden section method ${ }^{8}$ and a simplex search method ${ }^{9}$ were utilized in the parameter estimation routine for single and multiple parameter searching. Using these routines, the computer searches for the best parameters in the prospective model to minimize the error between the simulation results and the experimental data. If a prospective model with the optimum parameters cannot produce a simulated biosensor output that fits the experimental data, another metabolic model may be chosen for additional computer simulation. This process can be continued until a satisfactory model is found. The process for parameter estimation and model building is demonstrated in Figure 9.

Since different parameters may have similar effects on the simulated current-time curve, different types of experimental data, which can distinguish some effects from the others, were used in a step-by-step manner to estimate certain parameters. First, the diffusivity of oxygen in the cell layer was estimated using the current-time curve of an electrode with immobilized, dead L1210 cells. This current-time curve was obtained by switching the electrode from an oxygen free solution to an oxygen-saturated solution. The estimated oxygen diffusivity, $D_{3}$, in the cell layer is $2.18 \times 10^{-5} \mathrm{~cm}^{2} / \mathrm{s}$. Since the gel-cell layer contained

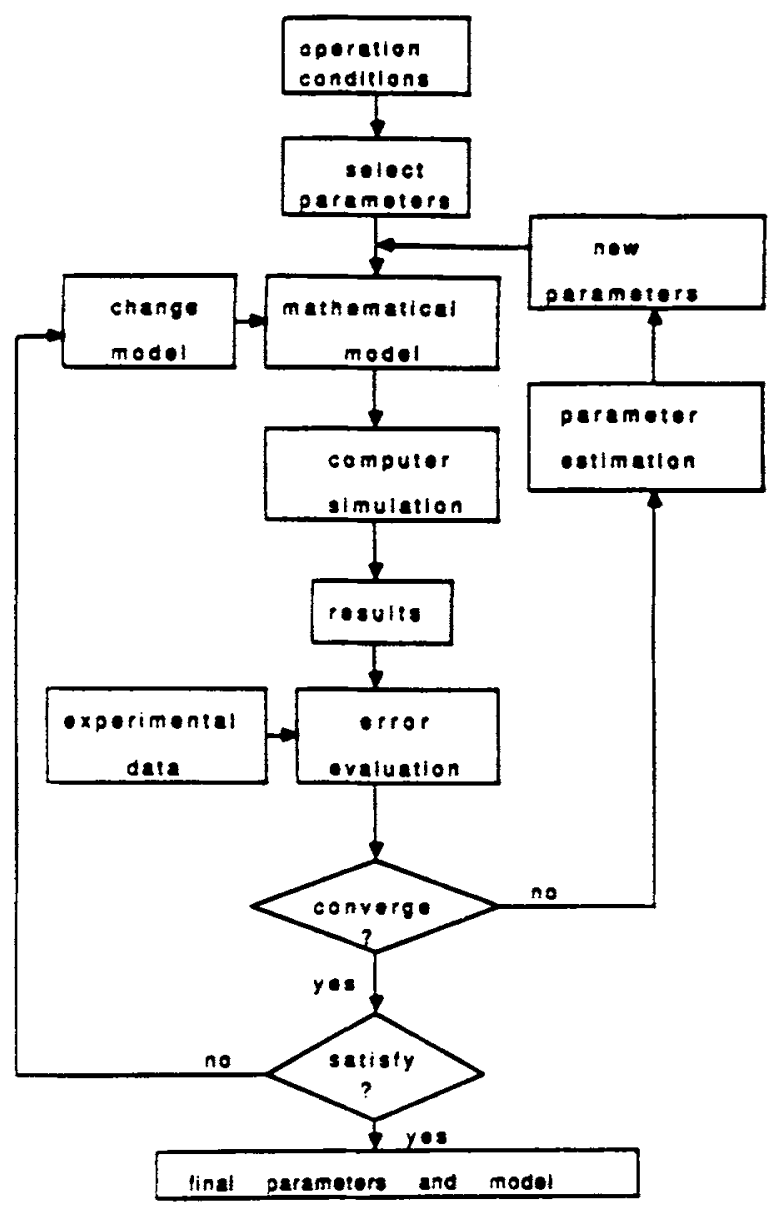

Figure 9. Parameter estimation and model building.

ca. $90 \%$ water, the estimated diffusivity is reasonable comparing to the oxygen diffusivity in water $\left(2.5 \times 10^{-5}\right.$ $\mathrm{cm}^{2} / \mathrm{s}^{10}$ ). The experimental current-time curve and the simulation results are plotted in Figure 10.

The respiration rate constants of $L 1210$, i.e., $\mu_{\max }$ and $k_{m}$, were estimated using the current-time curve of a biosensor with viable cells. A dynamic response was obtained by switching the biosensor from an oxygen free solution to an oxygen-saturated solution. The estimated $\mu_{\max }$ is $2.96 \times 10^{-18} \mathrm{gmol} / \mathrm{s} / \mathrm{cell}$, and the $k_{m}$ value is $1.47 \times$ $10^{-6} \mathrm{gmol} / \mathrm{mL}$. The simulation results and the data are compared in Figure 11.

In order to determine the suitable kinetic models for cell-chemical interaction between L1210 cells and Mechlorethamine $\mathrm{HCl}$, different kinetic models were used in the computer simulation and the parameter estimation. The results are plotted in Figures 12 and 13. The parameters obtained are also displayed in Figures 12 and 13. In Figure 12 , the simulation results are obtained using kinetic model (1) for chemical deactivation and kinetic model (1) for cell death. In Figure 13, the simulation results are obtained using kinetic model (2) for chemical deactivation and model (2) for cell death. When model (1) was used, the simulated current-time curve could not predict the lag phase and the trend of the current change. This is especially true for the lowest drug concentration. The current- 


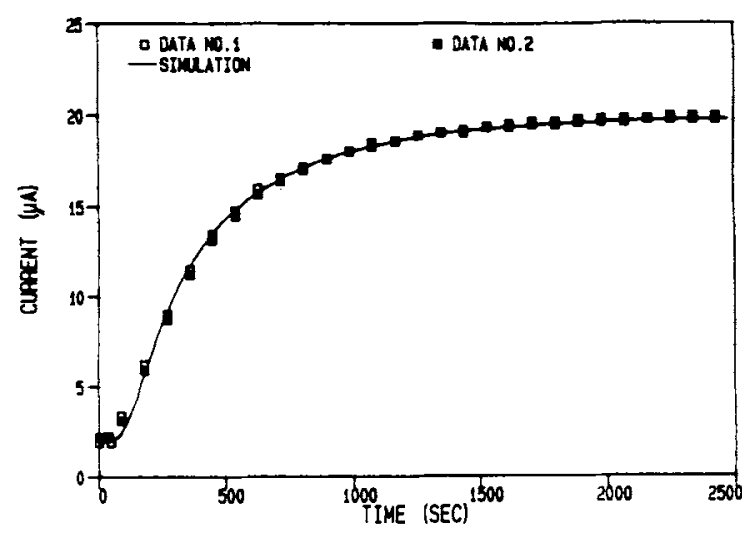

Figure 10. Dynamic response of biosensor with dead cells.

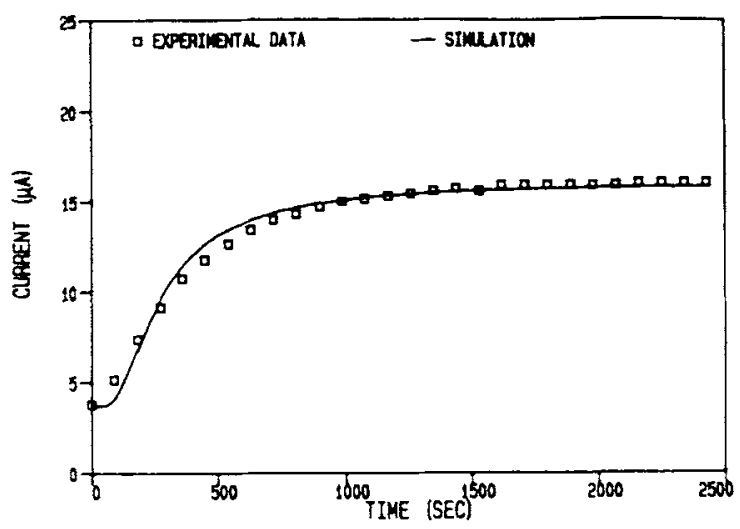

Figure 11. Dynamic response of biosensor with viable cells.

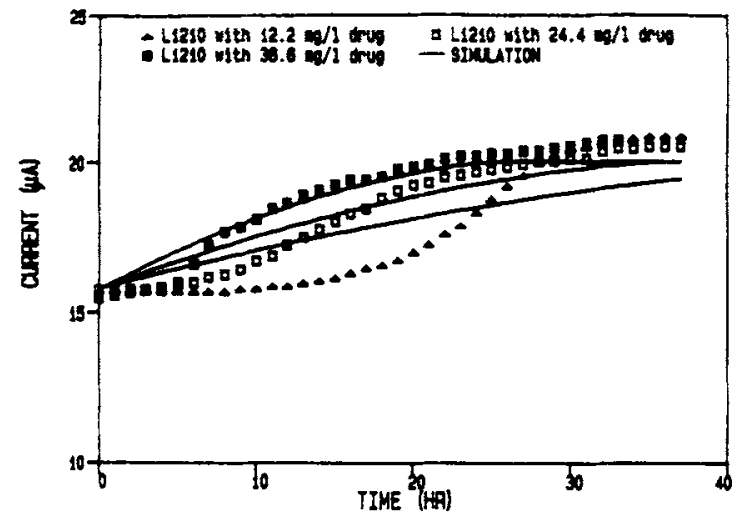

Figure 12. Response of $\mathrm{L} 1210$ biosensor to mechlorethamine $\mathrm{HCl}$ and computer simulation results using power law: $d N / d t=-k_{s} C^{n} N$; deactivation rate of drug $=k_{d} C^{n} N ; n=2 ; k_{s}=3.1 \times 10^{13} \mathrm{gmol}^{-2} / \mathrm{s} \mathrm{cm}^{6}$; $k_{d}=0.373 \mathrm{gmol}^{-1} \mathrm{~cm}^{6} / \mathrm{s} / \mathrm{cell}$.

time curve produced by model (1) was $\mathrm{C}$ shaped, but the experimental results were $S$ shaped. In such a case, human judgment should be used to choose another model. A general power law including the time variable, model (2), was then used in the computer simulation and parameter estimation. Using this model, the computer simulation reproduced the biosensor responses with reasonable agreement. This type of S-shaped response of the biosensor with L1210 was related to the drug-cell interaction mode. Mechlorethamine $\mathrm{HCl}$ causes breaks in replicating DNA strands. ${ }^{11}$ It is expected that this mode of interaction will cause a time-

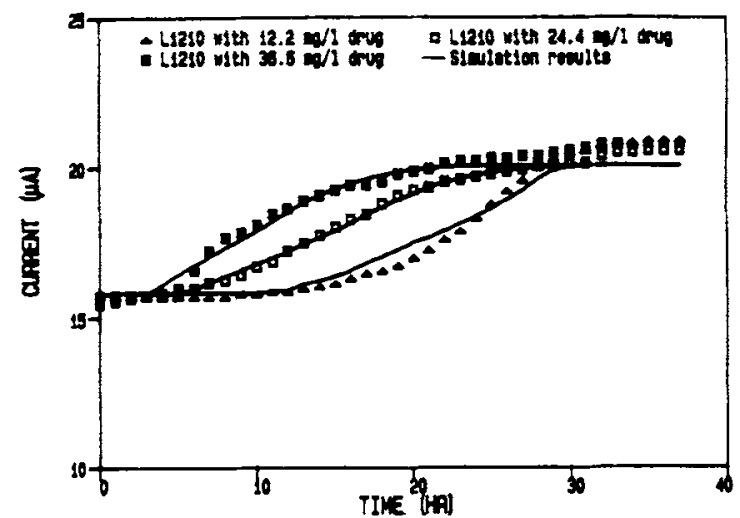

Figure 13. Response of $\mathrm{L} 1210$ biosensor to mechlorethamine $\mathrm{HCl}$ and the computer simulation results using: $d N / d t=-k_{s} C^{n} t^{m} N$ for $t x C>$ $T C_{0} ; d N / d t=0$ for $t x C<T C_{0}$; deactivation rate of drug $=k_{d} C^{n} N$; $n=2, m=0.32 ; k_{s}=7 . \times 10^{9} \mathrm{gmol}^{2} / \mathrm{s}^{-1.32} \mathrm{~cm}^{6} ; k_{d}=8.9 \times 10^{-3}$ $\mathrm{gmol}^{-1} \mathrm{~cm}^{6} / \mathrm{s} / \mathrm{cell} ; T C_{0}=8.77 \times 10^{-4} \mathrm{~s} \mathrm{gmol} / \mathrm{cm}^{3}$.

delayed response, and a S-shaped curve. The parameters for model (2), such as the rate constants and the product of critical time and concentration, can be utilized to characterize the cell-chemical interaction.

\section{CONCLUSIONS}

Based on our experimental and theoretical work, we can draw the following conclusions: biosensing is a promising technique for chemical testing and screening. By recording the biosensor output, the interaction between cells and chemicals can be monitored continually. With the appropriate hardware and software, the testing is amenable to automation. Different cell lines and various types of transducers can be used to produce a matrix for matching and testing. Using properly developed procedures, the biosensors can provide useful information about cell-chemical interactions.

The computer-aided analysis (CAA) system is useful for interpretation of the biosensor responses and model building in cell-chemical interaction studies.

\section{NOMENCLATURE}

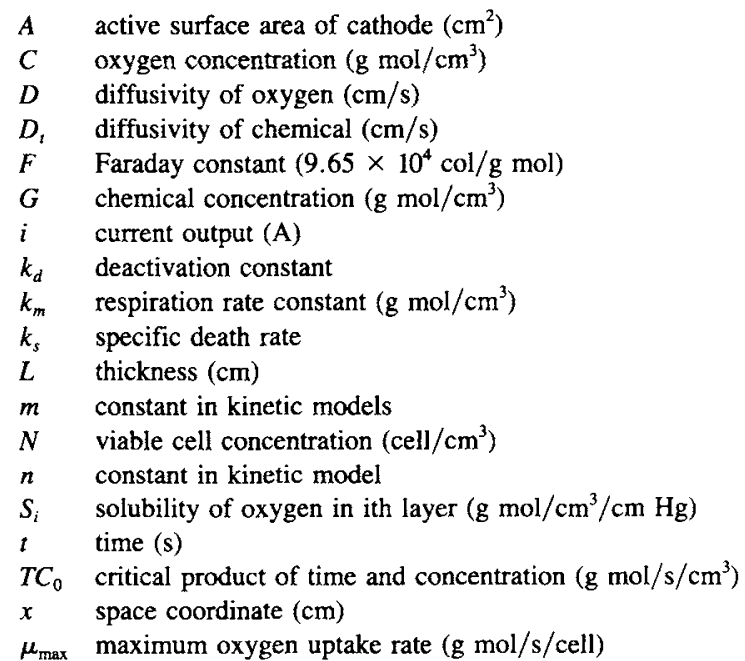


Subscripts

$i \quad i$ th layer

$s \quad$ bulk solution

$t \quad$ chemical

\section{References}

1. C. R. Lowe, Biosensors, 1, 3 (1985).

2. I. Karube, T. Matsunaga, and S. Suzuki, Anal. Chim. Acta, 109, 39 (1979).

3. G. A. Rechnitz, R. K. Kobos, S. J. Riechel, and C. R. Gebauer, Anal. Chim., 94, 357 (1977).

4. J.D. Borkowski and M. J. Johnson, Biotechnol. Bioeng., 9, 635 (1967).
5. B. S. Liang, X. M. Li, and H. Y. Wang, Biotechnol. Prog., 2, (4), 187 (1986).

6. X.M. Li, B.S. Liang, and H. Y. Wang, "Mathematical Modeling of Immobilized Cellular Electrodes", Proceedings of the 3rd Word Congress of Chemical Engineering, Tokyo, Japan, 1986, pp. 859-862.

7. A. Prokop and A. E. Humphrey, in Disinfection, M. A. Bennarde, Ed. (Marcel Dekker, New York, 1970), Chap. 3, p. 61.

8. W. E. Biles and J. J. Swain, Optimization and Industrial Experimentation (Wiley, New York, 1980), p. 188.

9. W. Spendly, G. K. Hext, and F. G. Himsworth, Technometrics, 4, (4), 441 (1962).

10. R. H. Perry and C. H. Chilton, Chemical Engineers' Handbook, 5th ed. (McGraw-Hill, New York, 1973), pp. 3-225.

11. R. T. Dorr and W. L. Fritz, Cancer Chemotherapy Handbook (Elsevier, New York, 1980). 